\title{
Numerical Analysis of a Pulse Detonation Cross Flow Heat Load Experiment
}

\author{
Daniel E. Paxson* \\ NASA Glenn Research Center, Cleveland, Ohio, 44130 \\ Andrew G. Naples ${ }^{\dagger}$ and John L. Hoke* \\ Innovative Scientific Solutions Inc., Dayton, $\mathrm{OH}, 45440$ \\ Fred Schauer ${ }^{\S}$
}

Air Force Research Laboratory, Propulsion Directorate, Wright-Patterson AFB, OH, 45433

\begin{abstract}
A comparison between experimentally measured and numerically simulated, timeaveraged, point heat transfer rates in a pulse detonation (PDE) engine is presented. The comparison includes measurements and calculations for heat transfer to a cylinder in crossflow and to the tube wall itself using a novel spool design. Measurements are obtained at several locations and under several operating conditions. The measured and computed results are shown to be in substantial agreement, thereby validating the modeling approach. The model, which is based in computational fluid dynamics (CFD) is then used to interpret the results. A preheating of the incoming fuel charge is predicted, which results in increased volumetric flow and subsequent overfilling. The effect is validated with additional measurements.
\end{abstract}

\section{Nomenclature}

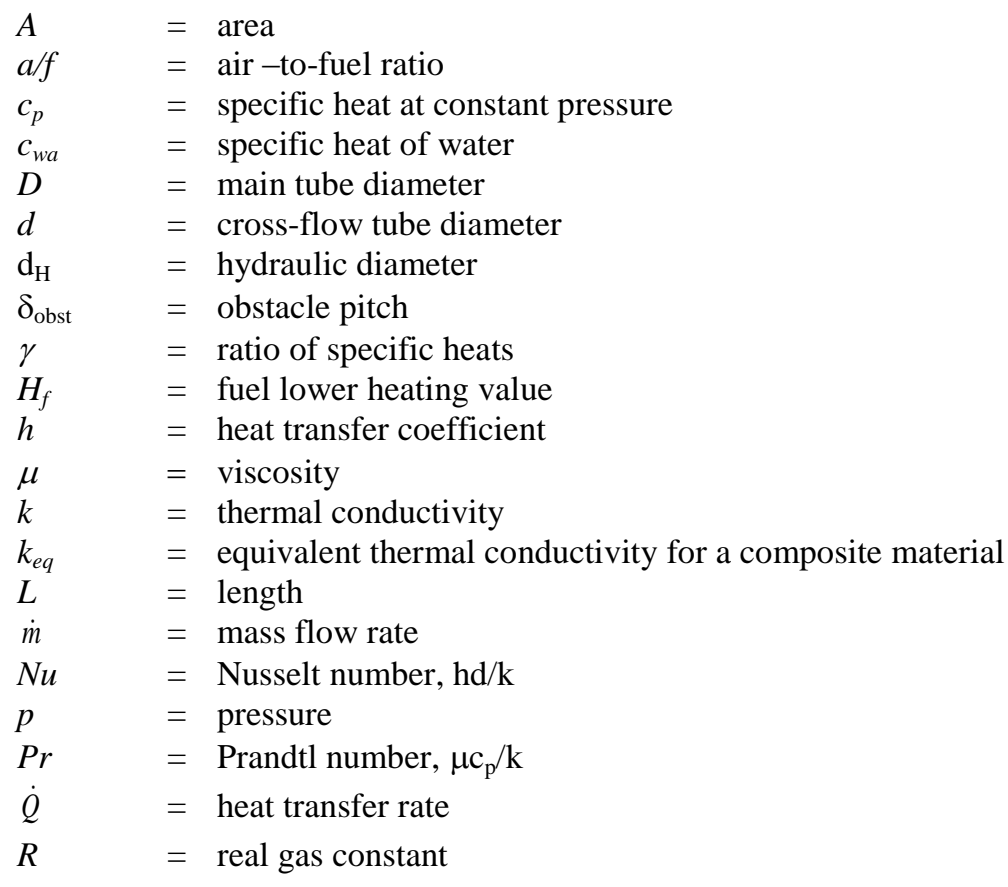

\footnotetext{
* Aerospace Research Engineer, 21000 Brookpark Road/MS 77-1,Cleveland, OH, 44135, AIAA Associate Fellow

${ }^{\dagger}$ Research Engineer, 2766 Indian Ripple Rd., Dayton, OH 45440, AIAA Member

* Senior Engineer, 2766 Indian Ripple Rd., Dayton, OH 45440, AIAA Senior Member

${ }^{\S}$ Research Engineer, 1790 Loop Rd., Dayton, OH 45433, AIAA Senior Member
} 


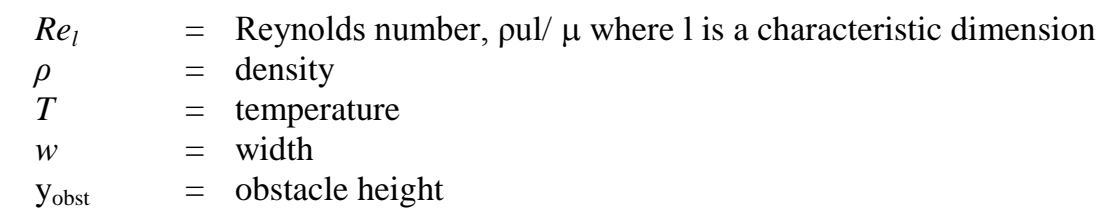

Subscripts:

$\begin{array}{ll}c & =\text { cross-flow tube } \\ c h & =\text { channel } \\ g & =\text { gas } \\ \text { ring } & =\text { annular ring } \\ \text { tube } & =\text { main tube wall } \\ w & =\text { wall } \\ w a & =\text { water } \\ d & =\text { cross flow tube diameter } \\ D & =\text { main tube diameter } \\ d_{H} & =\text { hydraulic diameter }\end{array}$

Superscripts:

$* \quad=\quad$ reference conditions used for normalizing data

\section{Introduction}

$\mathrm{P}$ rediction and management of thermal loads in detonation based propulsion systems is a critical technology development area for advancing their practical use. Despite its importance however, relatively few studies have been published this area ${ }^{1-7}$. Experimentally, this may be partly due to the difficulty in obtaining heat transfer measurements in the harsh environment of pulse detonation engines (PDE's). Analytically, it may relate to the formidable task of numerically simulating the heat transfer process. This conjugate problem requires knowledge of both the dynamics of the working fluid, and the thermal dynamics of the solid into which heat is being transferred.

It is possible to simplify the numerical problem by employing the concept of the heat transfer coefficient, along with the bulk properties of the fluid and solid. There is a clear loss of fidelity and/or accuracy in doing so; however, as long as suitable correlations for the heat transfer coefficients can be developed, it does make the problem manageable. This approach was used with some success in the work of Ref 8 . Here, the simulated performance impact (i.e. reduced thrust) of deflagration-to-detonation (DDT) enhancing obstacles correctly matched experimental results from long duration tests run to thermal equilibrium. Analysis of the numerical results showed that the reduction was largely due to heat transfer effects, which can be summarized as follows: a) relatively hot walls preheat the incoming fresh charge resulting in spillage of detonable fluid (overfilling from increased volumetric flow); b) the preheated charge in the tube is less dense and therefore results in a lower post-detonative, thrust producing pressure; c) relatively cold walls cool the hot, post-detonative gas thereby reducing useful work extraction. It is noted however that, although the effects just mentioned were indicated by the simulation results, and although the simulation correctly predicted thrust, there was no definitive proof that the heat transfer rates were correct, or that any of the effects mentioned were actually occurring.

In 2009 however, an experiment was conducted at the Air Force Research Laboratory in which time averaged heat transfer was measured on a cross-flow tube placed within, but near the exit of, the main tube of a PDE ${ }^{9}$. The setup was then reconfigured such that the cross-flow tube was exposed to steady, deflagrative combustion products, at the same overall equivalence ratio and averaged flow rates as the PDE. This was done by effectively removing the controlling valves of the PDE, and installing a flameholding device upstream of the cross-flow tube.

The surprising result of this experiment was that the detonative heat loads were less than those from deflagration. The logical conclusion drawn was that, while heat transfer rates are substantial during the actual detonation process, the detonation is of such short duration that the total heat transferred with each cycle is relatively small.

From the perspectives of analysis, simulation, and prediction of PDE heat transfer rates, this experiment represented an excellent opportunity to validate the model approach just mentioned ${ }^{8}$. This paper describes such an effort. The experiment and simulation will first be described, albeit briefly since they have been detailed in previous publications. The assumed heat transfer path of the crossflow tube will be presented next, along with the correlations used to obtain heat transfer coefficients. Predicted heat flux will then be compared to measured values. 
The initial validation effort led to further experimental testing and simulation work, which will also be presented. The crossflow tube was replaced with a spool piece capable of measuring main tube wall heat transfer rates, which are more relevant to PDE performance. Modification details will be shown. Simulated and measured results will then be compared.

The results will show that the correlated heat transfer coefficient approach to modeling heat transfer effects is reasonably accurate. Furthermore, it will show that heat transfer can substantially alter the operation of a PDE by preheating the incoming flow, subsequently causing spillage, reducing thrust, and reducing total heat transferred.

\section{Experimental Rig Description}

The experimental results are obtained from the PDE at the Air Force Research Laboratory's Pulsed Detonation Research Facility ${ }^{10-11}$. This PDE uses the head and valves of an automotive engine to control the airflow into the detonation tube. The PDE cycle consists of equal time allotted for: i) filling the detonation tube with pre-mixed ethylene and air at an equivalence ratio of one, ii) ignition, detonation, and blow-down, and iii) purging of the detonation tube with air. The pressure upstream of the automotive poppet valves is controlled such that, during the fill cycle, the mass flow of pre-mixed fuel and air is equal to that required to fill the tube to a specified fraction (the fill fraction) of its volume, at the upstream gas temperature and atmospheric pressure. Purge air (unfueled air which buffers incoming fresh charge from the residual hot gases of the previous cycle) is controlled in a similar fashion, and is quantified with a similar purge fraction. The fuel-air mixture is ignited with a $115 \mathrm{~mJ}$ spark at the valved end.

Up to four tubes can be sequentially fired on the rig. The frequency of operation, the fill fraction, and the purge fraction can all be varied, along with fuel to air ratio, the fuel type, and the spark delay. Numerous measurements are available from the rig, including thrust, and average flow rate. Additionally, the rig can accommodate tubes of varying length and diameter. For this work, measurements were made on only one tube, and thrust was not measured.

The experimental setup is shown schematically in Fig. 1. Water was run through the 0.25 in. o.d. cross-flow tube at a known rate during PDE operation. The temperature change across the cross-flow tube was measured. With the known specific heat of water, the cycle averaged heat transfer rate into the tube could be calculated from the following equation.

$$
\dot{Q}=\dot{m}_{w a} c_{w a}\left(T_{o u t}-T_{\text {in }}\right)
$$

The measured heat transfer rate changed (increased) from the start to the finish of each test run. The values used for the present study were those obtained at the end of runs lasting 7.5 minutes, when thermal equilibrium had nearly been reached, and heat transfer rate readings were nearly steady.

\section{Simulation Description}

Details of the simulation used in this study are given in Ref. 8. As such, only a brief description is presented here. The simulation (a.k.a. model or code) uses a high-resolution, time-accurate, single progress variable, reactive, quasi-one-dimensional (Q1D) computational fluid dynamics (CFD) code which integrates differential equations of motion for a calorically perfect gas, thereby capturing the detailed gasdynamic phenomena occurring inside a tube of specified cross sectional area distribution.
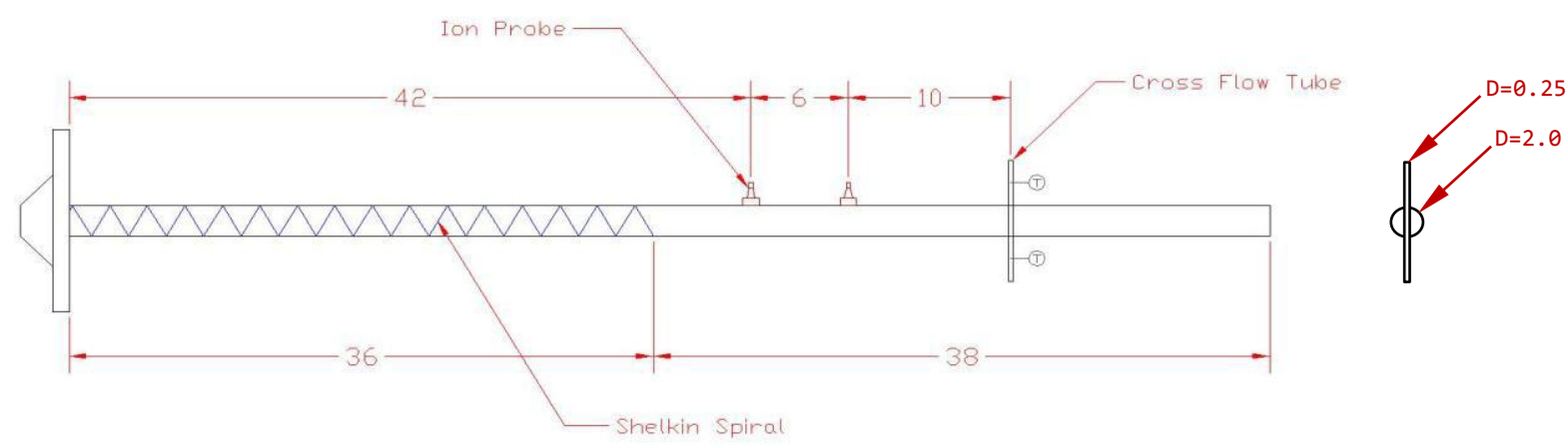

Figure 1 Schematic of the experimental setup. Dimensions are inches. 
Losses from viscous wall shear forces and heat transfer at the walls are incorporated as source terms with associated friction and heat transfer coefficient correlations that depend on local fluid velocity, density, and temperature. Separate correlations are employed for regions with and without DDT obstacles (e.g. the Schelkin spiral shown in Fig. 1). Heat transfer is assumed to occur between the working fluid and the passage walls only (i.e. no axial conduction along the tube, and negligible heat transfer on the outside of the tube). Each wall section corresponding to a numerical cell is assumed to maintain a fixed temperature over the course of one detonative cycle. The net heat transferred to or from the wall is monitored over the course of the cycle. When each cycle is complete, the wall temperature is updated via simple Euler integration. The detonative cycle is then repeated with the updated wall temperature. This process continues until the net heat transfer from each numerical wall section is zero.

The one-step reaction mechanism of the code is relatively simple. As long as there is reactant present, a prescribed temperature threshold has been reached, and the non-dimensional reaction rate constant is high enough, detonation will occur. Initiation and deflagration to detonation details cannot be captured with this mechanism; however, this is not considered a drawback for the present work.

Boundary conditions are supplied to the code as total conditions at the inlet, and static conditions at the exit. The conditions are used, along with characteristic equations, to determine appropriate states for "ghost" numerical cells which lie just outside the computing domain at each end of the tube. Ambient static pressure is imposed at the exit; however, a logic structure exists within the code such that when the exit flow is supersonic (and cannot sustain a normal shock), the imposed pressure is neglected and exit conditions are appropriately extrapolated from the interior. A logic structure also exists such that if the imposed static pressure cannot support outflow, the pressure is treated as a total value and, together with a supplied total temperature, is used to determine an appropriate inflow state.

A permanently open boundary condition is maintained at the head end of the tube; however, the pressure and temperature for this boundary are supplied by a coupled, lumped-volume, well-stirred reactor sub-model of the cylinder head which comprises the actual head end of the engine. Inflow rates of premixed detonable mixture, or purge air are prescribed (temporally and quantitatively) to match those of the rig. Outflow from the sub-model at any given instant of time is equal to the inflow predicted by the head-end numerical cell of the CFD code. Thus, the state in the sub-model depends on flow quantities from the CFD code, while the flow into the CFD code depends on states predicted by Euler integrated lumped volume sub-model. Hence, they are coupled

In order to crudely mimic the initiation process which follows spark ignition in the actual rig, the head-end reaction rate is set several orders of magnitude lower than the value used by the CFD code in the tube.

Generally, the reaction is not allowed to proceed unless the temperature is above a prescribed threshold value. However, in order to initiate the reaction at a desired 'spark' time, the threshold criterion is temporarily removed.

For all of the results to be presented, 200 numerical cells were used. The time step was approximately $3 \mu$ sec. Other relevant simulation parameters are listed in Table 1.

\section{A. Cross-Flow Tube Model}

The posited paths of heat transfer in the cross-flow tube are illustrated in Fig. 2. The code just described was used to compute the fluid state and velocity, as a function of time, at the axial location corresponding to the cross flow tube. This data was in turn used to estimate a (time dependent) heat transfer coefficient for the outer wall of the cross flow tube. The estimate was based on a standard correlation, and may be written as ${ }^{12}$

$$
N u_{d_{-} g}=0.25 \operatorname{Re}_{d_{-g}}^{0.6} \operatorname{Pr}_{g}^{0.8}
$$

Table 1 Simulation Parameters

\begin{tabular}{|l|l|}
\hline$R_{g}, \mathrm{ft}-\mathrm{lb}_{\mathrm{f}} / \mathrm{lb}_{\mathrm{m}} \cdot \mathrm{R}$ & 53.6 \\
\hline$\gamma$ & 1.31 \\
\hline$H_{f}, \mathrm{BTU} / \mathrm{lb}_{\mathrm{m}}$ & 20,250 \\
\hline$a / f)_{\text {stoichiometric }}$ & 14.8 \\
\hline$p^{*}, \mathrm{psia}$ & 14.7 \\
\hline$T^{*}, \mathrm{R}$ & 520 \\
\hline $\bar{\mu}_{g}, \mathrm{lb}_{\mathrm{m}} / \mathrm{ft} \cdot \mathrm{s}$ & $2.5 \mathrm{E}-5$ \\
\hline $\bar{k}_{g}, \mathrm{ft}-\mathrm{lb}_{\mathrm{f}} / \mathrm{ft} \cdot \mathrm{s} \cdot \mathrm{R}$ & 0.008 \\
\hline $\operatorname{Pr}_{g}$ & 0.70 \\
\hline
\end{tabular}

Here, the Nusselt number and Reynolds number are defined respectively as $N u_{d}=\frac{h_{g-w} d}{k_{g}}$, and $R e_{d}=\frac{\rho_{g} u_{g} d}{\mu_{g}}$, where $h_{g-w}$ is the gas-to wall heat transfer coefficient, $k_{g}$ is the gas thermal conductivity, $d$ is the diameter of the cross flow tube and $\rho_{g}, u_{g}$, and $\mu_{\mathrm{g}}$ are the gas density, velocity, and viscosity. Values for $k_{g}$ and $\mu_{g}$ were curve fit as functions of $T_{g}$ using available gas property tables ${ }^{13}$. The term $P r_{g}$ in Eq. 1 refers to the gas Prandtl number, and was fixed at a value of 0.7 . The cycle-averaged heat transfer rate from the gas to the wall of the cross flow tube is then written as

\section{4}

American Institute of Aeronautics and Astronautics 


$$
\dot{Q}_{g-w}=\pi L_{c}\left(\overline{N u_{d_{-} g} k_{g} T_{g}}-\overline{N u_{d_{-} g} k_{g}} T_{w}\right)
$$

In this equation $L_{c}$ is the cross flow tube length and $T_{g}$ and $T_{w}$ are the gas and wall temperatures.

A steady heat transfer coefficient for the inner cross flow tube wall surface may also be found in Ref. 12 as

$$
N u_{d_{-} w a}=0.023 \operatorname{Re}_{d_{-} w a}^{0.8} \operatorname{Pr}_{w a}^{0.4}
$$

The subscript $w a$ in this equation refers to the properties of the water. Values of $k_{w a}$ and $\mu_{w a}$ (as well as $C_{w a}$ in Eq. 1) are assumed constant. This correlation can be used to find the average heat transfer rate from the cross flow tube wall to the water.

$$
\dot{Q}_{w-w a}=N u_{d_{-} w a} k_{w a} \pi L_{c}\left(T_{w}-\bar{T}_{w a}\right)
$$

Referring to Fig. 2, the average water temperature is $\bar{T}_{w a}=\frac{\left(T_{o u t}+T_{\text {in }}\right)}{2}$.

Given a known cooling water entry temperature of $T_{i n}=520 \mathrm{R}$., and noting that $\dot{Q}=\dot{Q}_{w-w a}=\dot{Q}_{g-w}$, Eqns. 1, 3, and 5 provide three equations which can be solved simultaneously for the three unknowns, $T_{w}, \dot{Q}$, and $T_{\text {out }}$.

\section{Initial Results}

Two test points were compared. Both had the following run parameters in common: fill fraction $=0.78$; purge fraction $=0.24$. In one case the operational frequency was $10 \mathrm{~Hz}$. In the other, it was 8 Hz. The fill fraction was chosen such that, at ambient conditions, the leading edge of the detonable slug would just reach the cross-flow tube when the fill process ended. The computed limit-cycle for the $10 \mathrm{~Hz}$. case is shown in Fig. 3. The plot shows color contours of normalized pressure, temperature, Mach number and reactant fraction (mass fraction of detonable mixture) inside the main tube over the course of one cycle. The reference conditions used for normalization are listed in Table 1 . Next to each contoured variable the highest and lowest value within the x-t space are listed. The

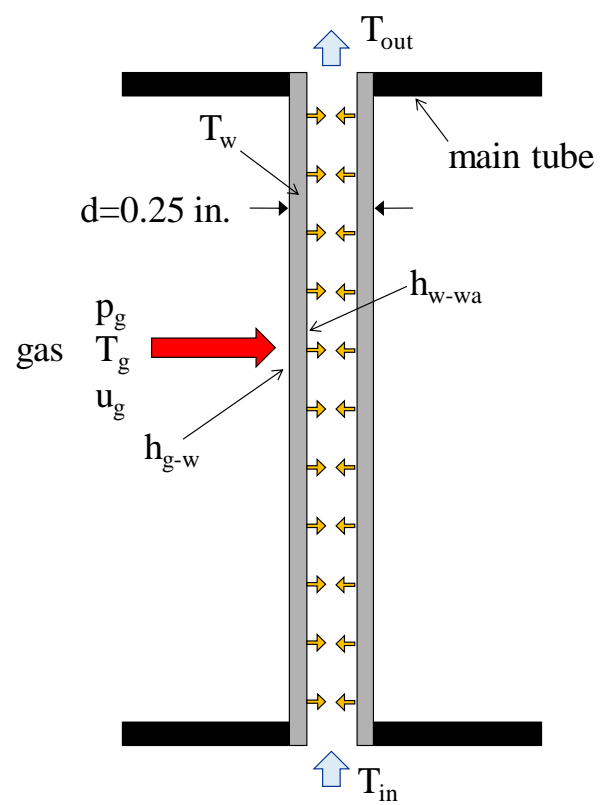

Figure 2. Heat flow paths for cross flow tube.

location of the cross-flow tube is shown as a white vertical line in the temperature and reactant fraction contours. Figure 3 illustrates several features relevant to the discussion to follow. It can be seen in the reactant fraction contour that the tube is substantially overfilling. This is caused by preheating from the hot tube walls, which can be observed in the temperature contours (note the axial gradient) during the filling stage of the cycle. It is noted, but not shown that when the computation is run adiabatically, the tube fills as prescribed. The result of this overfilling is that the cross-flow tube never encounters a significant fraction of the chemical energy entering the tube.

A final relevant feature of the flowfield shown in Fig. 3 can be seen in the temperature and Mach number contours. Here it is seen that there is a significant inflow period at the exhaust end of the PDE tube after the detonation occurs. During this period, cooler air is drawn past the cross-flow tube and may reduce the total heat transferred to the tube during the course of a cycle. Such flow reversals actually occur in many laboratory PDE's and have been measured ${ }^{14}$. They are not desirable, as they result from operating a device at low frequencies compared to the theoretical optimum. As such, they are not expected in practical PDE's. 


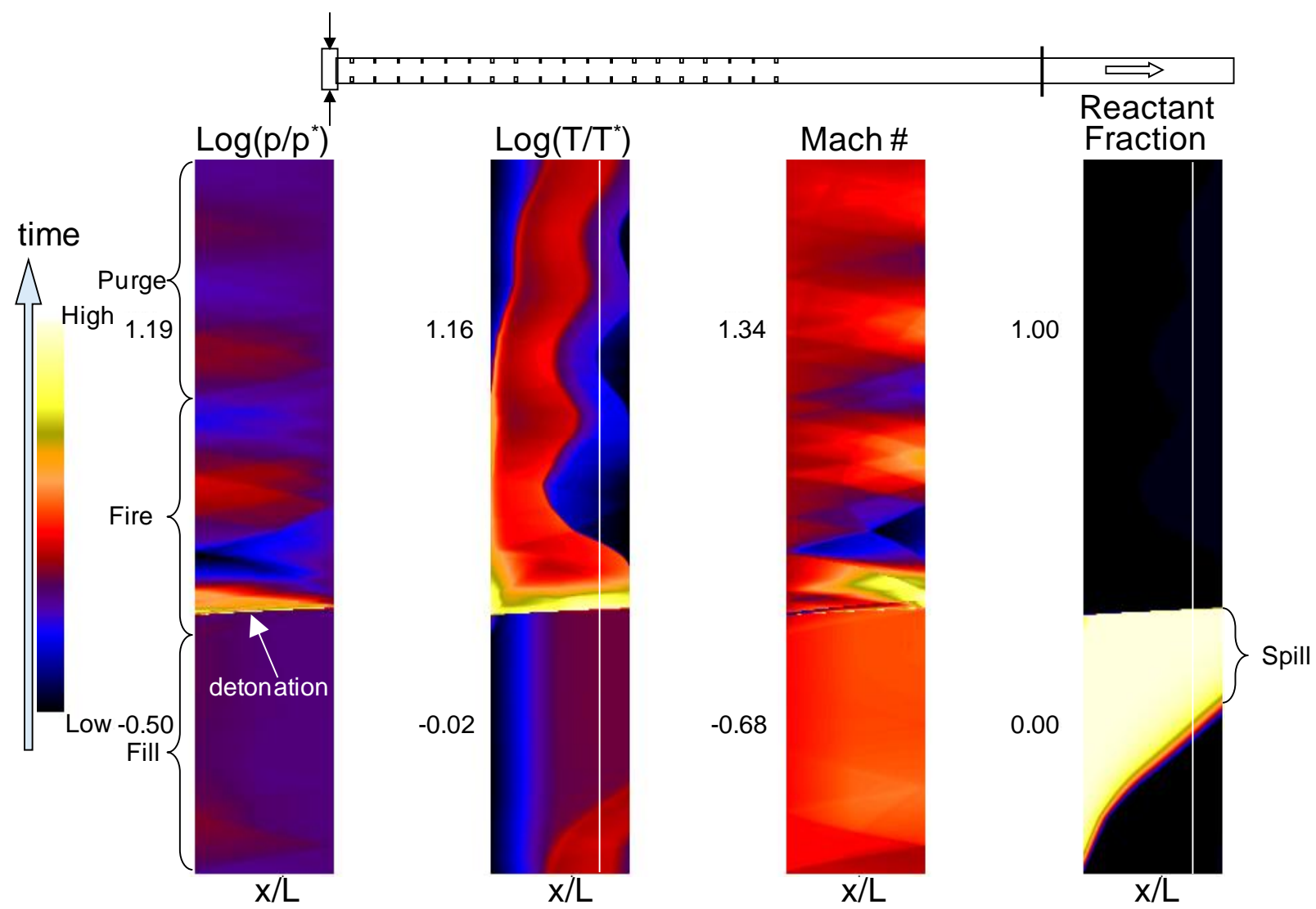

Figure 3. Computed limit cycle contours of normalized pressure, temperature, Mach number, and reactant fraction in the tube, over one period of the experimental rig operating at $10 \mathrm{~Hz}$. The white vertical line in the temperature and reactant fraction contour shows the location of the cross flow tube.

The instantaneous heat transfer rate to the cross-flow tube, computed from the simulated data and Eq. 3, is shown in Fig. 4 over the course of one cycle. The vertical scale has been expanded such that the heat transfer rate during fill and purge portion of the cycle can be seen; however, doing so cuts off visibility of the peak rates occurring during detonation. As such, the figure contains an insert showing the heat transfer rate during this period. Also shown in Fig. 4 are the computed and measured time-average heat transfer rates. It is worth noting that the peak heat transfer rates are more than two orders of magnitude above the average rate. The agreement between measured and computed averages is quite good. This is remarkable considering the simplicity of the model.

Results from the 8 and $10 \mathrm{~Hz}$. cases examined are summarized in Table 2. It is seen that for both cases, the simulated and measured heat transfer rates agree to within 6\%. Also shown in the table are estimated heat transfer rates for steady deflagrative combustion at the same overall equivalence ratio and flow rates (and in the same geometry) as the PDE cases. These estimates were made using the same Eqns. 1,3 , and 5 but with gas properties

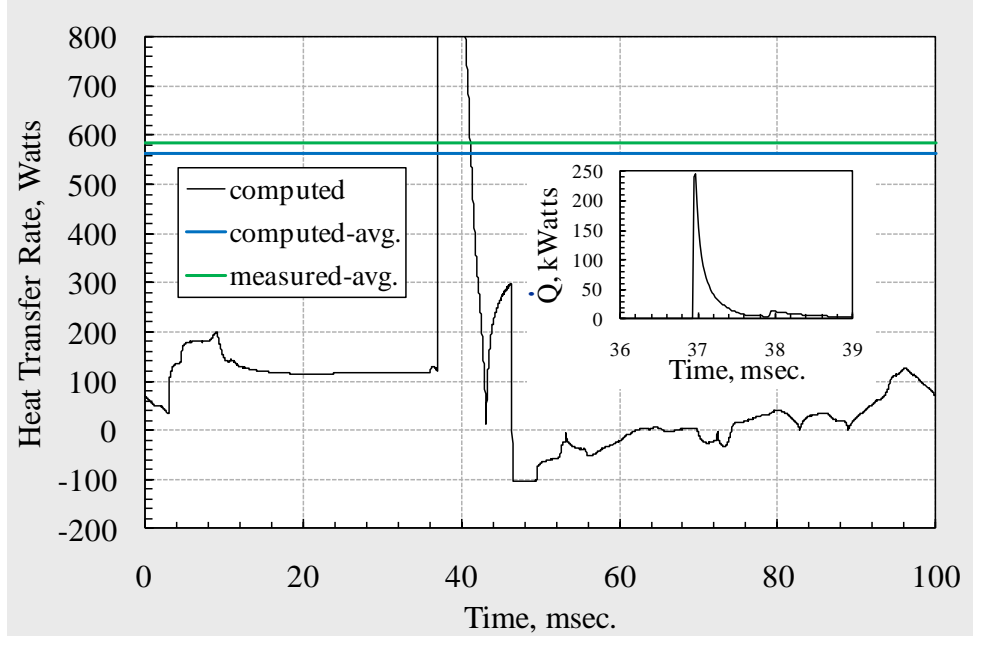

Figure 4. Computed heat transfer rate to the cross-flow tube as a function of time over the course of one $10 \mathrm{~Hz}$. cycle. corresponding to steady, post-deflagrative 
Table 2 Measured and simulated heat transfer rates.

\begin{tabular}{|c|c|c|c|c|c|}
\hline $\begin{array}{c}\text { Frequency } \\
\text { Hz. }\end{array}$ & $T_{w}$ & $\dot{Q}_{\text {measured }}$ & $\begin{array}{c}\dot{Q}_{\text {simulated }} \\
\text { Watts }\end{array}$ & $\begin{array}{c}\text { Difference } \\
\%\end{array}$ & $\begin{array}{c}\dot{Q}_{\text {deflagratin }} \\
\text { (estimated) } \\
\text { Watts }\end{array}$ \\
\hline 8 & 798 & 490 & 461 & -5.9 & 887 \\
\hline 10 & 858 & $585^{\dagger}$ & 562 & -3.9 & 991 \\
\hline
\end{tabular}

${ }^{\dagger}$ This test was only run for 2 minutes (not 7.5 minutes). The value listed in the table is extrapolated based on temporal trends of the longer $8 \mathrm{~Hz}$. run which show an approximately $30 \%$ increase in heat transfer rate from 2 to 7.5 minutes. combustion products. It is not meaningful to list the measured steady deflagrative heat transfer rates of the Ref. 9 experiment since the experimental rig exceeded acceptable metal temperature limits before thermal equilibrium was achieved. The estimates appear in Table 2 to emphasize the point that simulation of the experiment leads to the same result as the experiment itself, namely, detonative heat loads at this axial location

are less than those during deflagration. However, the simulation indicates that the effect is due largely to spillage, and not to the short duration of detonation event. In fact, the simulated results of the $10 \mathrm{~Hz}$. case indicate that approximately $37 \%$ of the flow passing the cross-flow tube is fueled. In terms of fuel utilized, this is roughly the equivalent of running with an overall equivalence ratio of 0.4 (the actual value is 0.76 ). If a steady, deflagrative equivalent to this run condition is calculated, the estimated heat transfer rate drops from 991 Watts to 376 Watts which is below the detonative heat load. Alternatively, though less realistically, if the detonative simulation is run with adiabatic PDE tube walls for the $10 \mathrm{~Hz}$. case, the average heat transfer rate to the cross-flow tube rises to 804 Watts. This is only $19 \%$ below the estimated load from deflagration. If $40 \mathrm{~Hz}$, adiabatic operation is simulated, along with more performance optimized valve timing, the disparity drops to only $9 \%$. If other, more practical cycle modifications were examined, it is quite possible that the disparity would vanish altogether. Considerably more investigation is required however before any conclusions can be drawn.

\section{Additional Testing and Simulation}

Although the cross-flow tube provided a convenient means of assessing heat loads, it was not clear that a cylinder in a cross-flow was representative of the true area of interest, the PDE tube wall. Moreover, since the simulated spillage and pre-heating described earlier were caused by heat transfer at the walls, it was important to assess the validity of the heat transfer correlations used. As such, a new test piece was constructed which operated on the same principle as the cross-flow tube, but had a decidedly different geometry. The piece is shown schematically in Fig. 5. A photograph of the piece is shown in Fig. 6. Referring to Fig. 5, it is seen that the analysis of this piece, heretofore called the spool, is similar to the cross-flow tube. However, different dimensions and correlations must be used, and a new heat transfer path has been added via conduction through the Macor rings.

\section{A. Spool Modeling}

For the gas-to-wall heat transfer path, the
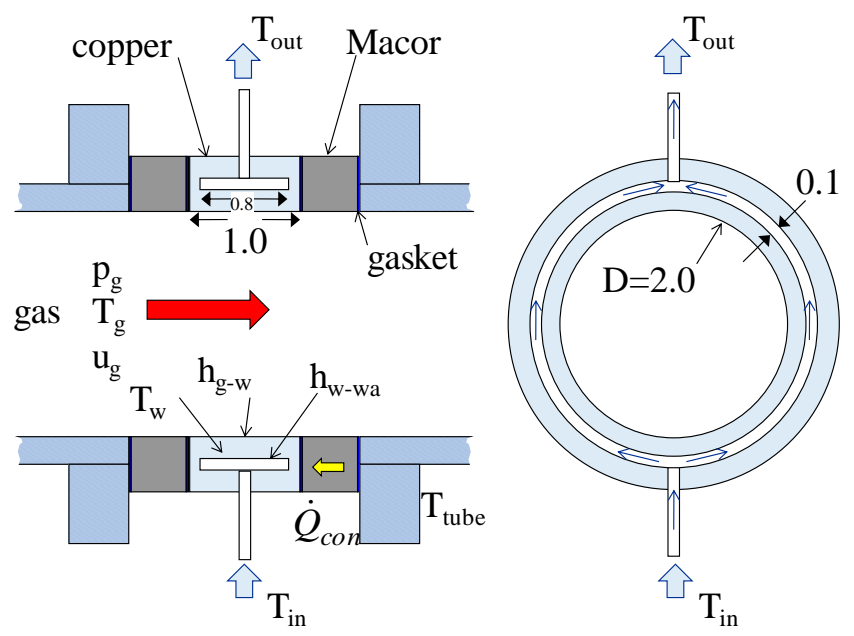

Figure 5. Schematic and heat flow path of the wall heat transfer spool. Dimensions are inches. correlation developed for the simulation was used ${ }^{8}$.

$$
N u_{D_{-} g}=0.121\left(\frac{D}{L}\right)^{0.352} \operatorname{Re}_{D_{-} g}^{0.80} \operatorname{Pr}_{g}^{0.33}
$$

The equation for the average heat transfer rate thus becomes

$$
\dot{Q}_{g-w}=\pi w_{\text {spool }}\left(\overline{N u_{D_{-} g} k_{g} T_{g}}-\overline{N u_{D_{-} g} k_{g}} T_{w}\right)
$$



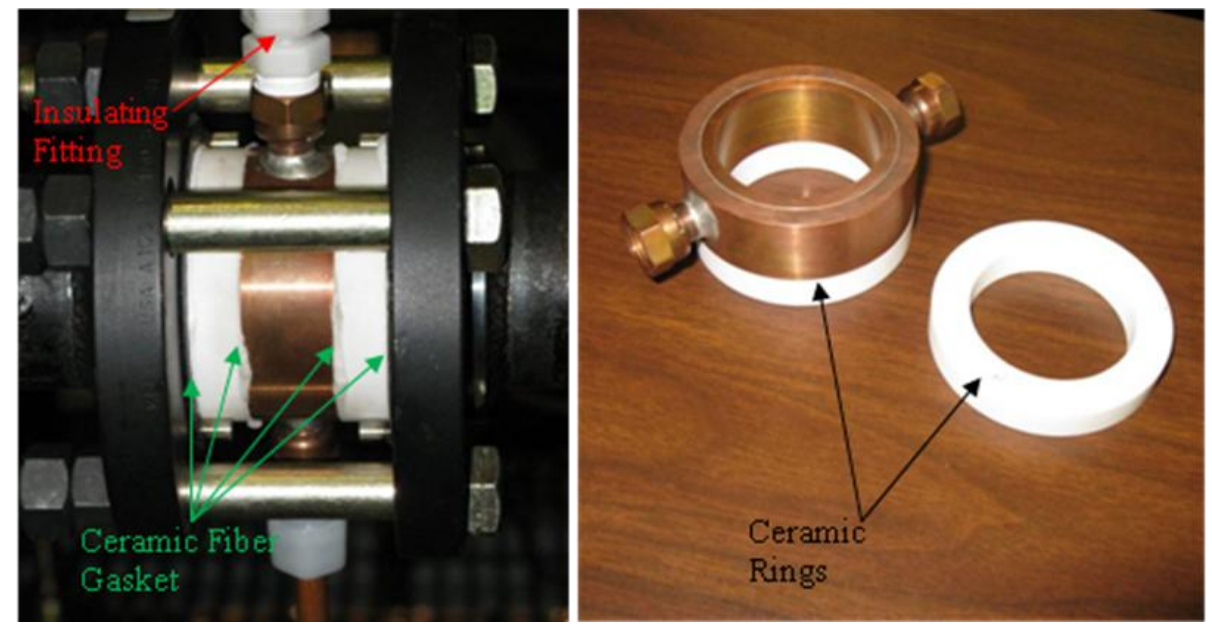

Figure 6: Photograph of wall heat transfer spool. Insulation was applied to the outside during testing

Here, $w_{\text {spool }}$ is the width (in the axial direction) of the spool. Since Eq. 6 was developed based on a calorically perfect gas, a single constant value for the viscosity was used in the gas Reynolds number. The same is true of the gas thermal conductivity used in Eq. 7. The values used are listed in Table 1.

The water flow in the channel was found to be laminar based on the calculated Reynolds number of 174 . As such, a laminar flow correlation with adjustment for entry length was used for the wall-to-water heat transfer coefficient $^{12}$.

$$
N u_{d_{H-w a}}=3.66+\frac{\left[.0668 \operatorname{Pr}_{w a}\left(\frac{d_{H}}{L_{c h}}\right) \operatorname{Re}_{d_{H} w a}\right]}{\left\{1+0.04\left[\operatorname{Pr}_{w a}\left(\frac{d_{H}}{L_{c h}}\right) R e_{d_{H} w a}\right]^{0.67}\right\}}
$$

The terms $d_{H}$ anfd $L_{c h}$ in this equation represents the hydraulic diameter and channel length respectively. The average heat transfer rate equation corresponding to Eq. 8 is

$$
\dot{Q}_{w-w a}=N u_{d_{H-w a}} k_{w a} 20.25 L_{c h}\left(T_{w}-\bar{T}_{w a}\right)
$$

The heat transfer rate due to the extra conduction path through the Macor rings is written as follows

$$
\dot{Q}_{\text {con }}=\frac{2 k_{e q} A_{\text {ring }}}{w_{\text {ring }}}\left(T_{\text {tube }}-T_{w}\right)
$$

Here, $k_{e q}$ is the equivalent thermal conductivity of the Macor ring and ceramic fiber gasket, $w_{\text {ring }}$ is the ring width, $A_{\text {ring }}$ is the ring contact area with the copper spool, and $T_{\text {tube }}$ is the temperature of the surrounding PDE tube wall. The value of $T_{\text {tube }}$ used was $1422 \mathrm{R}$. This represents a typical value obtained from the simulation by averaging the 200 computed wall temperatures when a limit cycle is reached. $T_{\text {tube }}$ varies depending on the operating condition simulated. The value used here is approximately midway between the highest and lowest value calculated in this work. Eq. 9 adds only one new unknown. Thus, together with the relation $\dot{Q}=\dot{Q}_{w-w a}=\dot{Q}_{g-w}+\dot{Q}_{c o n}$, closure of Eqs. 1, 7, 9, and 10 can be obtained. 


\section{Additional Results}

The spool was installed on the PDE at approximately the same location as the original cross-flow tube. The layout is shown schematically in Fig. 7. Also shown in this schematic are the locations of several ion gages which can be used to detect detonation. This will become relevant in the discussion to follow. The PDE was operated at 8 Hz., with a purge fraction of 0.23 . The fuel was again ethylene, and the equivalence ratio was again set to 1.0. Four 6.0 minute runs were made which maintained fill fractions of $0.75,0.60,0.45$, and 0.34 . The simulation was run to limit cycle operation for these same four test conditions.

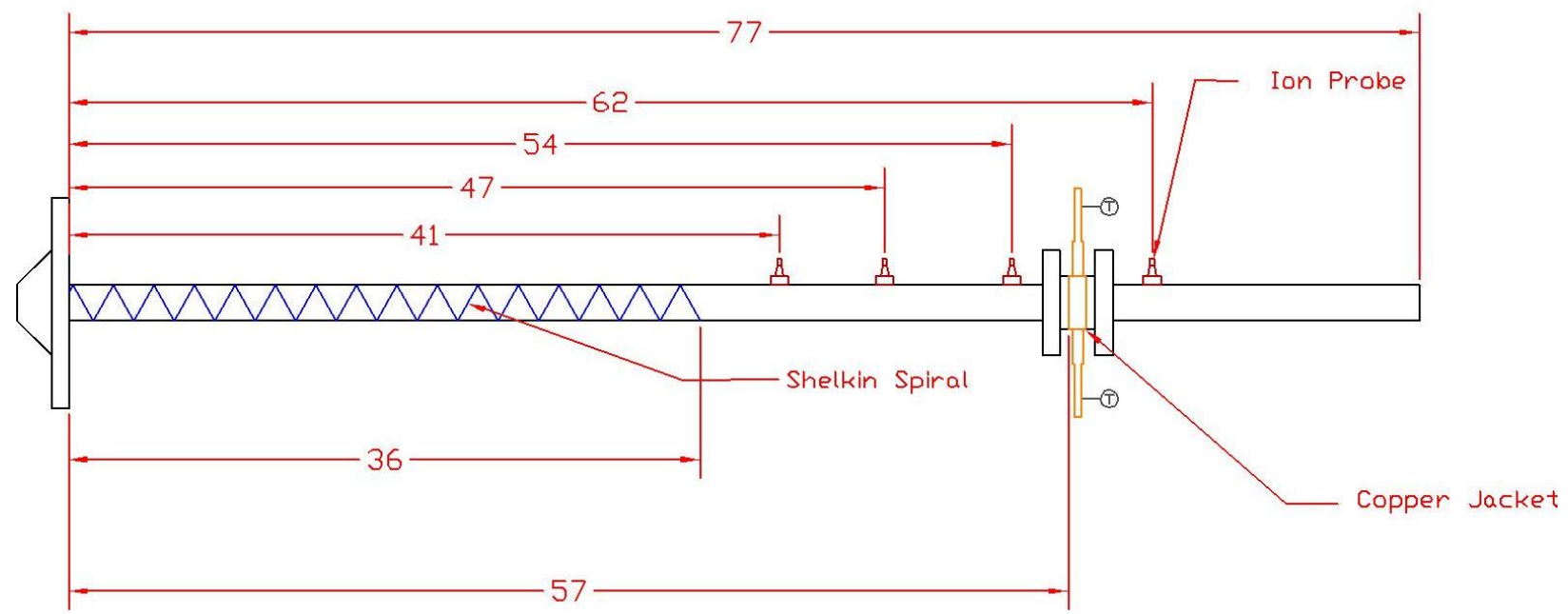

Figure 7 Schematic of the experimental setup for wall heat transfer testing. Dimensions are inches.

The measured and computed average total heat transfer rates are shown in Fig. 8 as functions of the fill fraction. The computed results also show contributions to the total heat transfer rate from conduction and convection. From a fill fraction of 0.45 to 0.75 , the agreement between computed and measured heat transfer is quite good. Below the 0.45 fill fraction, the computation appears to over-predict. It should be born in mind however, that the disparity at this point is $31 \%$, which is within the stated accuracy band of many heat transfer correlations ${ }^{12}$. Taken as a whole, these results seem to validate the heat transfer coefficient used in the simulation.

It is interesting to note that the computed convection heat transfer rate at a fill fraction of 0.75 is less than the $461 \mathrm{~W}$. value calculated for the cross-flow tube under the same PDE conditions. Given the relative surface areas of the two measuring systems, this result may seem at first surprising. However, cylinders in cross flow, with associated stagnation points, and regions of massive separation, generally have significantly higher heat transfer coefficients than surfaces aligned with the flow such as the spool.

The fact that the average heat transfer rate is non-linear with fill fraction suggests that pre-heating and overfilling is occurring. The simulation results indicate that even at the lowest fill fraction of 0.34 , the tube is actually volumetrically fully filled. Experimentally, the ion gages were monitored (though not recorded) during the entire run at this fill fraction. Initially, with cold tube walls, none of them registered a detonation. This would be expected since no preheating could occur and since the fill fraction was such that a detonation never passed an ion gage. As time progressed however, ion gages registered regular

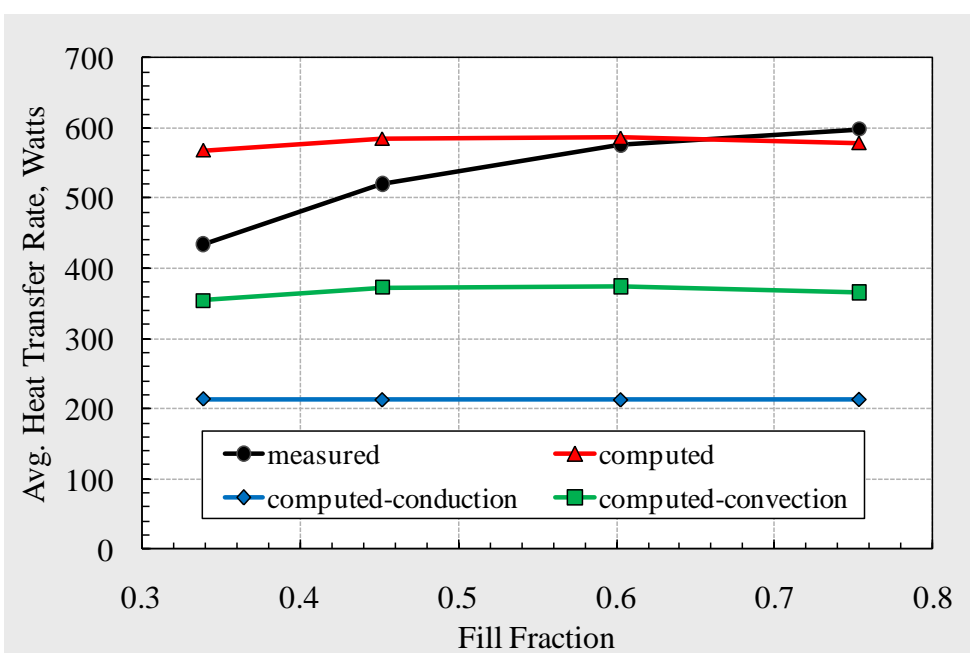

Figure 8. Measured and computed average wall heat transfer rates at $x / L=0.75$ as functions of fill fraction for $8 \mathrm{~Hz}$. operation. 
detonations further and further down the tube. At the conclusion of the test, the ion gage located $70 \%$ of the way down the tube was registering detonations. Even the gage at the $80 \%$ mark was registering a combustion event. This observation seems to validate the behavior predicted by the simulation (overfilling due to preheating), although clearly not to the same level.

Computed heat flux to the wall during the detonation phase of the fill fraction $=0.75,8 \mathrm{~Hz}$. limit cycle is plotted in Fig. 9. The peak value of $16 \mathrm{MW} / \mathrm{m}^{2}$ is of the same order as the $32 \mathrm{MW} / \mathrm{m}^{2}$ peak value reported from a high frequency response heat flux gage located near the exit of a single shot hydrogen/air PDE experiment ${ }^{7}$. Given the differences in the two experiments, the simplicity of the model, and the difficulty in obtaining such high and short-lived heat flux levels, this is considered acceptable agreement.

\section{A. Alternate Location}

As a final comparison test between

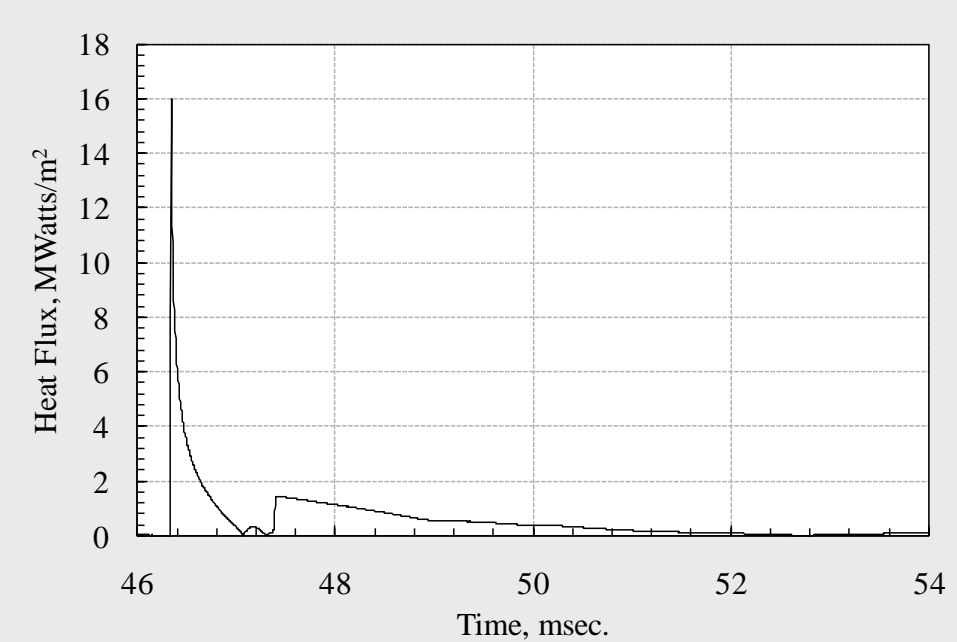

Figure 9. Computed wall heat flux at $x / L=0.75$ during the detonation period of an $8 \mathrm{~Hz}$., fill fraction $=0.75$ cycle. simulation and experiment, the spool was moved to a location further upstream in the PDE. The setup is shown in Fig. 10. This measurement location is somewhat problematic from a simulation standpoint because a different wall heat transfer coefficient correlation from Eq. 6 is employed for regions where DDT obstacles exist. The formula is as follows ${ }^{8}$.

$$
N u_{D_{-} g}=0.121\left(\frac{y_{\text {obst }}}{\delta_{\text {obst }}}\right) R e_{D_{-} g} \operatorname{Pr}_{g}^{0.33}
$$

Here, $y_{o b s t}$ and $\delta_{o b s t}$ are the obstacle height (or spiral diameter) and pitch respectively. This formula generally produces much higher heat transfer coefficients than Eq. 6, which is appropriate given the elevated turbulence levels and coherent flow structures produced by DDT obstacles. The problem with the location shown is that it is just 1.5 tube diameters downstream from end of the DDT obstacles. It would be expected therefore that the heat transfer rate would fall somewhere between the extremes predicted by Eqs. 6 and 11 .

Beyond this ambiguity issue, there is also the observation that in this region of the tube the detonation may still

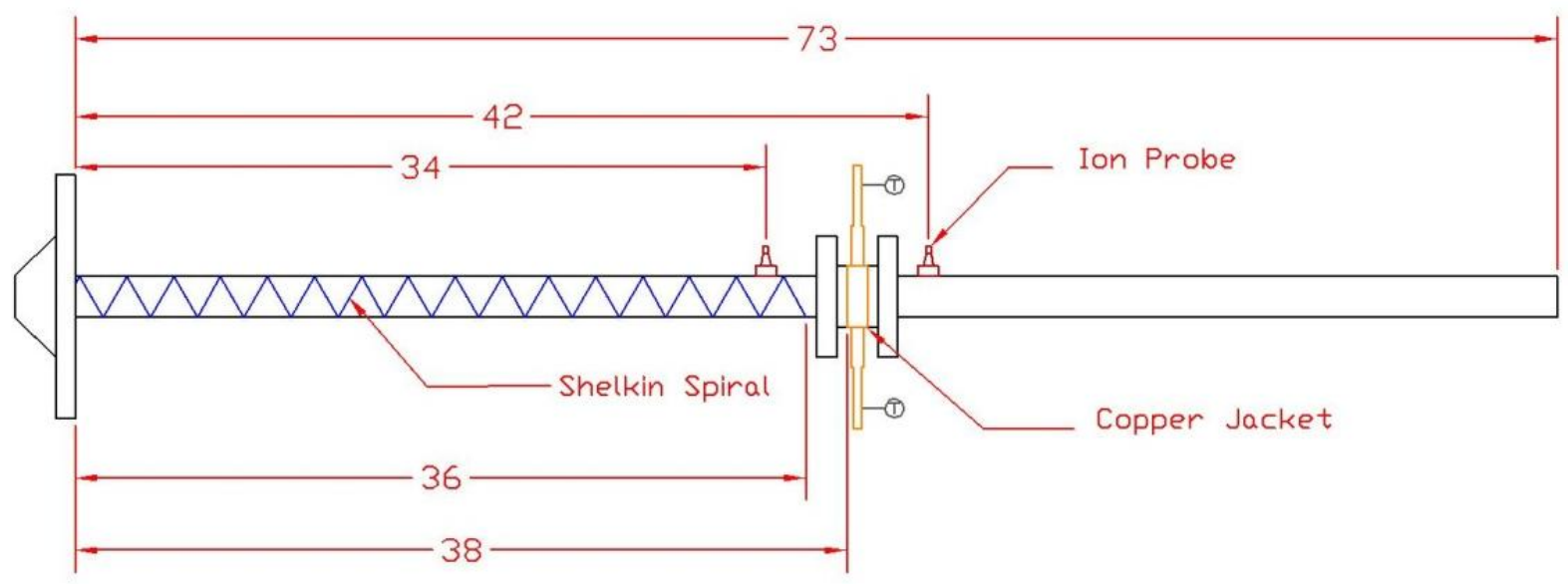

Figure 10 Schematic of the experimental setup for the upstream spool location. Dimensions are inches. 
be in the over-driven phase of development which would result in higher heat transfer rates. Such a phase is not modeled in the simulation.

Two runs were made in the Fig. 10 configuration. Both were made at $8 \mathrm{~Hz}$. operation. One utilized a fill fraction of 0.79 , the other utilized 0.4 . The measured and computed average heat transfer rates are shown in Fig. 11. For the computed rates, the results from using both Eq. 6 and Eq. 10 are shown. As expected, the measured results fall between the computed extremes. It is re-emphasized however, that the elevation of the measured results over the computed results using Eq. 6 may also be partially explained by the presence of an over-driven detonation (although the same axial location for the over-driven phase at two vastly different fill fractions is somewhat surprising). Ultimately resolving the cause will require, at the very least, heat transfer rate measurements at different locations and in different configurations. Nevertheless, it is encouraging that the simulation at least bounded the results.

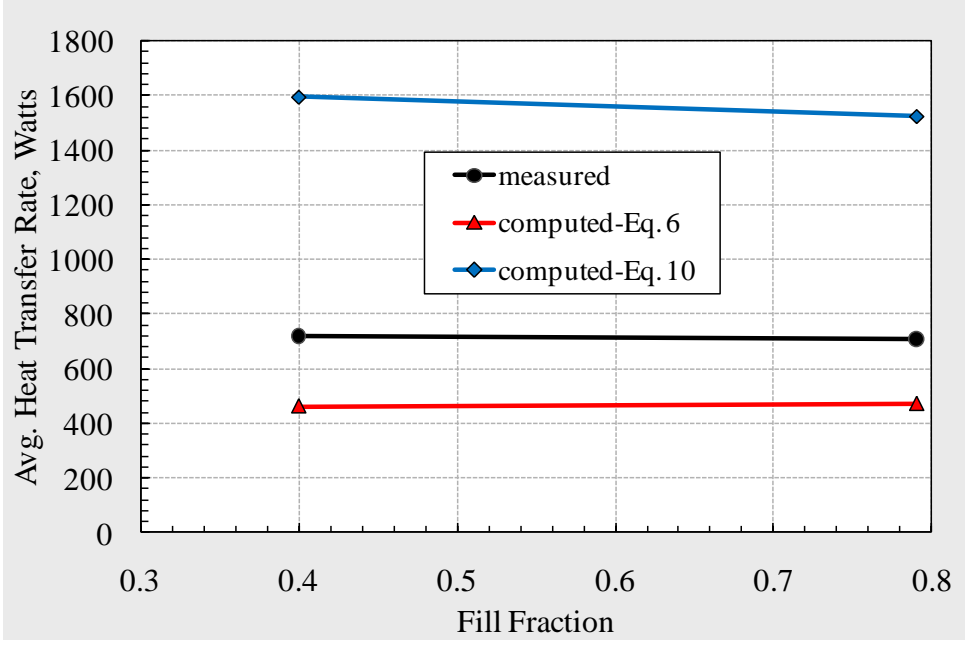

Figure 11. Measured and computed average wall heat transfer rates at $\mathrm{x} / \mathrm{L}=0.53$ as functions of fill fraction for $8 \mathrm{~Hz}$. operation.

\section{Concluding Remarks}

A comparison between experimentally measured and numerically simulated (computed), time-averaged, point heat transfer rates in a pulse detonation (PDE) engine was presented. The comparison included measurements and calculations for heat transfer to a cylinder in cross-flow and to the tube wall itself, using a novel spool design. Measurements were obtained at several locations and under several operating conditions. The measured and computed results were found to be in substantial agreement. The agreement served to validated the computational modeling approach. This suggests that such an approach can be used for the critical task of assessing the heat loads and thermal management requirements of for practical PDE applications. Validation of the computational modeling also provided a measure of confidence in the use of computational fluid dynamics (CFD) codes for interrogating experimental results to provide insight where instrumentation cannot. To this end, it was shown that, for the particular rig simulated, the operational impact of heat transfer is substantial. It was predicted, and later verified that the walls of the PDE tube, which are heated substantially during operation, in turn pre-heat the incoming detonable mixture. This results in increased volumetric flow rates during tube filling, which leads in turn to overfilling and fuel spillage. Knowledge of the degree to which this is occurring is invaluable in interpreting performance results (e.g. specific impulse) obtained during long duration operation.

\section{References}

${ }^{1}$ Hoke, J., R. Bradley, and F. Schauer. Heat Transfer and Thermal Management in a Pulsed Detonation Engine. in 41st AIAA Aerospace Sciences Meeting and Exhibit. AIAA 2003-852. Reno, NV.

${ }^{2}$ Eidelman, S., D. Sharov, and D. Book. Aerothermodynamics of Pulsed Detonation Engines. in 36th JANNAF Meeting. Cocoa Beach, FL.

${ }^{3}$ Ajmani, K. and K.J. Breisacher. Qualitative Study of Cooling Methods for a Pulsed-Detonation Engine. in 51st JANNAF Propulsion Meeting. Lake Buena Vista, Florida.

${ }^{4}$ Kasahara, J., K. Takazawa, and T. Arai. Experimental Study of Impulse and Heat Transfer on Pulse Detonation Engines. in 38th AIAA/ASME/SAE/ASEE Joint Propulsion Conference and Exhibit. AIAA 2002-4071. Indianapolis, IN.

${ }^{5}$ Bykovskii, F.A., Thermal Fluxes in Combustion Chamber Walls in the Detonation and Turbulent Combustion Modes. Fizika Goreniya i Vzryva, 1991. 27(1): p. 70-75.

${ }^{6}$ Ajmani, K., et al. Numerical and Experimental Studies of a Film Cooled Pulsed Detonation Tube. in 41st AIAA/ASME/SAE/ASEE Joint Propulsion Conference \& Exhibit. AIAA 2005-3509. Tucson, AZ.

${ }^{7}$ Paxson, D.E., Perkins, H. D., "Thermal Load Considerations for Detonative Combustion-Based Gas Turbine Engines," AIAA-2004-3396, July, 2004. 
${ }^{8}$ Paxson, D. E., Schauer, F. R., and Hopper, D. R., "Performance Impact of Deflagration to Detonation Transition Enhancing Obstacles," AIAA paper 2009-502, January, 2009.

${ }^{9}$ Naples, A. G., Hoke, J. L., Schauer, F. R., "Study of Heat Loads from Steady Deflagration and Pulsed Detonation Combustion," AIAA paper 2010-956, January, 2010.

${ }^{10}$ Schauer, F., J. Stutrud, and R. Bradley. "Detonation Initiation Studies and Performance Results for Pulsed Detonation Engine Applications," AIAA Paper 2001-1129, Jan., 2001

${ }^{11}$ Schauer, F.R., Bradley, R. P. and Hoke, J. L., "Detonation Initiation of Hydrocarbon-Air in a Pulsed Detonation Engine," AIAA-2005-1343, Jan., 2005.

${ }^{12}$ Holman, J. P., Heat Transfer, $5^{\text {th }}$ edition, McGraw-Hill, 1981, pp. 226-244.

${ }^{13}$ Gordon, S. and McBride, B., "Computer Program for Calculation of Complex Chemical Equilibrium Compositions and Applications," NASA RP-1311, Oct, 1994.

${ }^{14}$ Opalski, A. B., Paxson, D.E., Wernet, M. P., "Detonation Driven Ejector Exhaust Flow Characterization Using Planar DPIV," AIAA-2005-4379, July, 2005. 